\title{
Nuclei Isolation from Adult Mouse Kidney for Single- Nucleus RNA-Sequencing
}

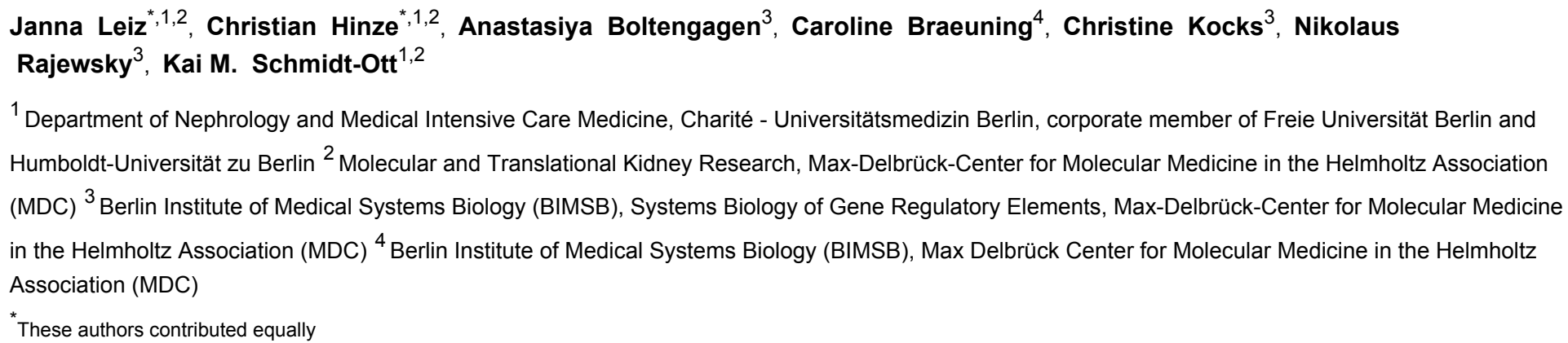

\section{Corresponding Authors}

Nikolaus Rajewsky

rajewsky@mdc-berlin.de

Kai M. Schmidt-Ott

kai.schmidt-ott@charite.de

\section{Citation}

Leiz, J., Hinze, C., Boltengagen, A., Braeuning, C., Kocks, C., Rajewsky, N., Schmidt-Ott, K.M. Nuclei Isolation from Adult Mouse Kidney for Single-Nucleus RNA-Sequencing. J. Vis. Exp. (175), e62901, doi:10.3791/62901 (2021).

\section{Date Published}

September 20, 2021

DOI

$10.3791 / 62901$

URL

jove.com/video/62901

\section{Abstract}

The kidneys regulate diverse biological processes such as water, electrolyte, and acid-base homeostasis. Physiological functions of the kidney are executed by multiple cell types arranged in a complex architecture across the corticomedullary axis of the organ. Recent advances in single-cell transcriptomics have accelerated the understanding of cell type-specific gene expression in renal physiology and disease. However, enzyme-based tissue dissociation protocols, which are frequently utilized for single-cell RNA-sequencing (scRNA-seq), require mostly fresh (non-archived) tissue, introduce transcriptional stress responses, and favor the selection of abundant cell types of the kidney cortex resulting in an underrepresentation of cells of the medulla.

Here, we present a protocol that avoids these problems. The protocol is based on nuclei isolation at $4{ }^{\circ} \mathrm{C}$ from frozen kidney tissue. Nuclei are isolated from a central piece of the mouse kidney comprised of the cortex, outer medulla, and inner medulla. This reduces the overrepresentation of cortical cells typical for wholekidney samples for the benefit of medullary cells such that data will represent the entire corticomedullary axis at sufficient abundance. The protocol is simple, rapid, and adaptable and provides a step towards the standardization of single-nuclei transcriptomics in kidney research.

\section{Introduction}

Kidneys display a highly complex tissue architecture. They along a corticomedullary axis and mediate biological consist of functionally and anatomically distinct segments 
functions, such as regulation of extracellular fluid volume, electrolyte balance, or acid-base homeostasis ${ }^{1}$.

Advances in single-cell transcriptomics have enabled the indepth characterization of complex tissues and accelerated the understanding of segment and cell type-specific gene expression in renal physiology, development, and disease $2,3,4$.

However, the enzyme-based dissociation protocols that are frequently utilized for scRNA-seq display several drawbacks and constraints. Depending on the protocol, they generate transcriptional stress responses and tissue dissociation bias towards easier-to-dissociate cortical cell types ${ }^{5,6}$. Although protocols using cold-active proteases for embryonic kidneys are able to mitigate stress-related transcriptional alterations, they fail to overcome the dissociation bias towards cortical cells and might not be readily adaptable to different kinds of diseased kidney tissues ${ }^{7}$. In addition, single-cell approaches are not easily compatible with frozen tissue samples, limiting their application mostly to non-archived, fresh tissue, thus making the tissue collection a restricting factor ${ }^{6}$.

Single-nuclei RNA sequencing (snRNA-seq) can circumvent these limitations $s^{8,9}$. Here, we present a protocol for nuclei isolation from a central slice of frozen adult mouse kidney tissue (Figure 1$)^{10}$. Our protocol is simple and provides a standardized approach to obtain RNA sequencing libraries with a balanced representation of diverse kidney cell types for experimental models that do not involve strong regional tissue changes. In the latter case, our protocol can also be performed with whole kidneys.

\section{Protocol}

All animal experiments were conducted in accordance with the Animal Welfare Act (TierSchG) and the Animal Welfare
Experimental Animal Regulation (TierSchVersV) and were authorized by local authorities and the Animal Welfare Officers at our institution (MDC).

\section{Tissue preparation}

1. Prepare a 6 -well plate containing $2 \mathrm{~mL}$ of $1 \mathrm{x}$ phosphatebuffered saline (PBS) per well for each kidney that will be obtained. Prepare a 6-well plate containing $2 \mathrm{~mL}$ of RNA stabilization solution per well and kidney. Pre-cool both plates on ice.

2. Euthanize a 3- to 6-month-old male C57BL/6 mouse. Place the mouse on a dissecting tray, pin down the extremities and sterilize the abdomen with $70 \%$ ethanol.

3. Open the abdomen up to the ribcage using forceps and scissors. Lift the intestine and other organs to the side and remove the kidneys by carefully cutting the ureter, renal artery, and vein with a scissor. Wash the kidney in the previously prepared, ice-cold 1x PBS and remove the renal fascia and any remaining fat from the kidney until all white tissue is removed (Figure 2A).

4. Place the kidney on a cold dissecting plate and use a sharp scalpel or razor blade to obtain a middle slice of 1-2 $\mathrm{mm}$. Make sure the tissue piece contains the entire corticomedullary axis (Figure 2B). Use microdissection scissors and forceps to carefully trim the cortex from the sides of the center piece. Within the dissected tissue piece, the three segments cortex, outer medulla, and inner medulla should be clearly visible (Figure 2C).

NOTE: The slice should not exceed a thickness of $2 \mathrm{~mm}$ or a weight of $20 \mathrm{mg}$ to ensure sufficient buffer amounts for effective tissue lysis and in order to minimize ambient background RNA in the cDNA libraries. Ambient RNA 
wastes sequence capacity, as it is not associated with single nuclei.

5. Transfer the kidney piece to the previously prepared RNA stabilization solution and incubate for $24 \mathrm{~h}$ at $4{ }^{\circ} \mathrm{C}$ to avoid RNA degradation. After $24 \mathrm{~h}$, remove the RNA stabilization solution and store the tissue at $-80{ }^{\circ} \mathrm{C}$ until further use. Carefully remove the excess solution with tissue paper.

\section{Nuclei isolation}

1. Cleaning and preparation steps

1. Clean benchtops and pipettes with $70 \%$ ethanol and RNase decontamination solution.

2. Clean a round-bottomed, $2 \mathrm{~mL}$ tissue grinder tube and matching pestle $A$ and $B$ with RNase decontamination solution, followed by $70 \%$ ethanol and RNase-free water (1 grinder tube and pestle set per sample). Let it dry completely.

3. Pre-cool the centrifuge to $4{ }^{\circ} \mathrm{C}$.

4. Label and pre-cool three $15 \mathrm{~mL}$ collection tubes, a $1.5 \mathrm{~mL}$ collection tube, a $5 \mathrm{~mL}$ fluorescenceactivated cell sorting (FACS) collection tube, and a dry grinder tube for each sample on ice.

\section{Buffer preparation}

1. Warm up the Ribonucleoside-vanadyl complex stock solution to $65{ }^{\circ} \mathrm{C}$ until reconstituted to a greenblack clear solution according to manufacturer's instructions. $^{11}$

2. Prepare $1 \mathrm{x}$ PBS containing 4\% bovine serum albumin (BSA) as described in Table 1A. Additionally, prepare 1x PBS with $0.04 \%$ BSA (Table 1B). Filter both solutions using a $0.2 \mu \mathrm{m}$ surfactant- free cellulose acetate (SFCA) membrane syringe filter and keep on ice until further use.

3. Prepare Nuclei Lysis Buffer 1 (NLB1, Table 1C). Add $4 \mathrm{~mL}$ of EZ lysis buffer for Nuclei Lysis Buffer 2 (NLB2, Table 1D) and $2 \mathrm{~mL}$ of $0.04 \%$ BSA / PBS for the Nuclei Suspension Buffer (NSB, Table 1E) to 15 $\mathrm{mL}$ tubes. Add the RNase inhibitor solution to NLB2 and NSB directly before use as indicated below in the protocol. Keep on ice until further use.

4. Prepare EZ lysis buffer with $10 \%$ sucrose (Sucrose Gradient Buffer, Table 1F). Mix well and filter the buffer into a fresh $15 \mathrm{~mL}$ tube using a $0.2 \mu \mathrm{m}$ SFCA membrane syringe filter. Keep on ice until further use.

\section{Tissue homogenization and cell lysis}

NOTE: In order to minimize RNA degradation, all steps are carried out on ice. The grinder tube, Petri dish, and all buffers need to be pre-cooled. All resuspension steps are done by carefully pipetting the nuclei suspension. Do not vortex the sample to avoid shearing forces and damage to nuclei.

1. Take the frozen kidney piece and transfer it to a 60 $\mathrm{mm}$ polystyrene Petri dish on ice containing $1 \mathrm{~mL}$ of NLB1.

2. Mince the tissue thoroughly using a razor blade or scalpel (Figure 3A).

3. Cut off the tip of a $1 \mathrm{~mL}$ pipette tip and transfer the minced tissue and buffer to the grinder tube. Make sure to transfer all tissue pieces. Wash the Petri dish 5-10 times with the buffer, if necessary.

4. Homogenize the suspension on ice by slowly moving pestle $A, 25 x$ up and down in the grinder tube. Avoid air bubbles caused by rapid movement (Figure 3B). 
5. Pass the homogenate through a $100 \mu \mathrm{m}$ strainer in a pre-cooled $15 \mathrm{~mL}$ collection tube and wash the filter with another $1 \mathrm{~mL}$ of NLB1.

6. Wash the grinder tube with cold EZ nuclei lysis buffer and discard the buffer.

7. Transfer the homogenate back into the grinder tube and homogenize the suspension on ice by slowly moving pestle $B, 15 x$ up and down in the grinder tube. Avoid air bubbles caused by rapid movement (Figure 3C).

8. Transfer the homogenate to a pre-cooled $15 \mathrm{~mL}$ collection tube. Wash the grinder tube with another $2 \mathrm{~mL}$ of NLB1 and make sure to transfer all tissue fragments to the collection tube. Incubate the homogenate (total volume of $4 \mathrm{~mL}$ ) for $5 \mathrm{~min}$ on ice to lyse the cells.

\section{Nuclei purification}

1. Pass the homogenate through a $40 \mu \mathrm{m}$ strainer into a pre-cooled $15 \mathrm{~mL}$ collection tube. Spin the collection tube for $5 \mathrm{~min}$ at $500 \times \mathrm{g}$ at $4{ }^{\circ} \mathrm{C}$ in a centrifuge with a swinging-bucket rotor. In the meantime, add RNase inhibitor solution to NLB2 (Table 1D).

2. Remove the supernatant without disturbing the pellet. Carefully resuspend the pellet in $4 \mathrm{~mL}$ of NLB2.

3. Carefully underlay the suspension with a $1 \mathrm{~mL}$ cushion of Sucrose Gradient Buffer. Centrifuge at $500 \times g$ for $5 \mathrm{~min}$ at $4{ }^{\circ} \mathrm{C}$ in a centrifuge with a swinging-bucket rotor. In the meantime, add RNase inhibitor solution to NSB (Table 1E).
4. After centrifugation, gently remove the collection tube from the centrifuge and be careful not to disturb the two layers when handling the collection tube. Cell debris is visible between the two layers. Remove the supernatant carefully starting with the debris. Remove the remaining supernatant without disturbing the nuclei pellet and carefully resuspend the pellet in $1 \mathrm{~mL}$ of NSB.

NOTE: The resuspension volume depends on the amount of tissue used for the isolation and pellet size gained after the last centrifugation step. The volume might need to be adapted to the expected number of nuclei.

5. Pass the homogenate through a $20 \mu \mathrm{m}$ strainer into the pre-cooled $5 \mathrm{~mL}$ FACS collection tube.

\section{Nuclei sorting}

1. Add $20 \mu \mathrm{L}$ of 4',6-diamidino-2-phenylindole (DAPI) per $\mathrm{mL}$ of NSB to a final concentration of $2 \mu \mathrm{M}$ to the homogenate in the FACS collection tube and mix carefully. Incubate for $5 \mathrm{~min}$ on ice.

2. Prepare the pre-cooled $1.5 \mathrm{~mL}$ collection tube with 20 $\mu \mathrm{L}$ of $4 \%$ BSA /1x PBS and add $0.5 \mu \mathrm{L}$ RNase inhibitor solution to a final concentration of $1 \mathrm{U} / \mu \mathrm{L}$. Immediately proceed to sorting.

3. Sort the nuclei using a cell sorter.

1. Mix the nuclei suspension briefly before inserting the FACS collection tube into the sorter.

2. Set a first gate P1 based on the forward scatter (FSC) and side scatter (SSC) to exclude debris and aggregates (Figure 4A).

3. To exclude empty or damaged nuclei and multiplets, set a subsequent gate based on the DAPI-Area 
versus DAPI-Height (DAPI-A vs DAPI-H) (Figure 4B).

4. Sort single nuclei into the $1.5 \mathrm{~mL}$ collection tube containing 4\% BSA /1x PBS with $1 \mathrm{U} / \mu \mathrm{L}$ RNase inhibitor solution prepared in 3.2 .

\section{Quality control}

1. Measure the final nuclei concentration under a fluorescence microscope or in an automated counting chamber in at least two independent counts and assess the suspension quality (Figure 5).

NOTE: Optimal concentrations are between 700 - 1,200 nuclei/ $\mu \mathrm{L}$. Lower cell concentrations such as 700 nuclei/ $\mu \mathrm{L}$ may be preferable as resulting cDNA libraries contained less ambient background RNA (transcripts not associated with individual nuclei).

2. Calculate the required volume of nuclei suspension for the desired recovery of sequenced single nuclei. In order to avoid nuclei aggregation and RNA degradation, proceed immediately to library preparation.

\section{Representative Results}

To determine the performance of our protocol, we used the 10x Genomics Chromium Single Cell 3' Gene Expression Kit v3.1 for library preparation and analyzed the snRNA-seq data with the Seurat package ${ }^{12,13}$.

Figure 6 shows results from a representative snRNA-seq library. To assess the quality of our nuclei, we plotted the number of genes against the number of transcripts (defined by unique molecular identifiers (UMIs)) colored by the fraction of mitochondrial reads (Figure 6A). Nuclei of good quality generally show higher numbers of reads, correlating UMI and gene numbers, and low mitochondrial read fractions.
For the subsequent analysis, nuclei with less than 500 or more than 4000 counted genes, or more than $5 \%$ of mitochondrial RNA were excluded $(n=828)$. Only genes expressed in a minimum of three nuclei were included. We detected about 20,000 genes in total in the remaining 6,000 nuclei with 1,600 median genes and 2,800 median UMls per nucleus (Figure 6B).

Clustering was based on highly variable genes. We identified a total of 18 clusters. Cell identities were annotated based on known marker genes (not shown). Subclusters of one cell type were summarized to one cluster resulting in a total of 11 distinct cell types: podocytes, proximal tubule (PT), thin limb (tL), thick ascending limb (TAL), distal convoluted tubule (DCT), connecting tubule (CNT), collecting duct principal and intercalated cells (CD-PC, A-IC, B-IC), deep medullary epithelium of the pelvis (DMEP) and endothelium. Gene expression patterns of cluster-enriched markers were visualized in a dot plot (Figure 6C) and cell type clusters in a t-distributed stochastic neighbor embedding (t-SNE) plot (Figure 6D).

To assess cell type distributions in our sample, the percentage of each cell type was calculated (Figure 6E) and used to determine the ratio of PT to TAL. The PT is mainly located in the kidney cortex and frequently overrepresented in kidney single-cell datasets as cells of the PT are easy to dissociate and highly abundant in whole kidney samples. The TAL on the other hand stretches across the whole outer medulla ${ }^{14}$. Thus, the ratio of PT and TAL fractions represents a good measure for the enrichment of medullary cell types in a kidney single-cell dataset. In general, the PT/TAL ratio in single-cell whole kidney datasets ranged from 8 (unpublished data from cold-protease-treated whole kidney tissue) to 45 for enzymatically dissociated tissue $10,14,15$. In the snRNA- 
seq dataset presented here we were able to reach a PT/TAL ratio of 2. This result illustrates that removal of excess cortex during tissue dissection combined with snRNA-seq results in a strikingly improved kidney cell type representation.

\section{Tissue dissection ( $\sim 30 \mathrm{~min})$}
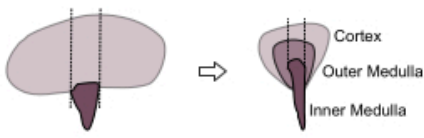

Middle slice dissection and cortex trimming (see dashed lines above)

\section{Nuclei sorting ( $230 \mathrm{~min})$}

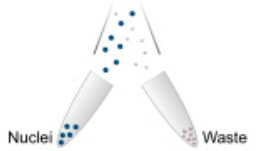

Cell debris and aggregate reduction
2. Nuclei isolation $(\sim 1 \mathrm{~h})$

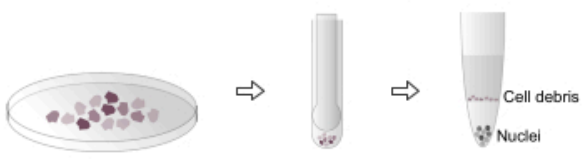

Tissue mincing, homogenization and sucrose gradient centrifugation

4. Quality control ( 10 min)
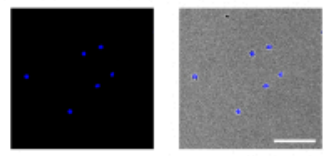

Nuclei quality and concentration assessment

Figure 1: Schematic overview of the workflow. The protocol consists of four major steps that include tissue dissection followed by nuclei isolation, nuclei sorting and a final purity and concentration assessment. Scale bar $=100 \mu \mathrm{m}$. Please click here to view a larger version of this figure.
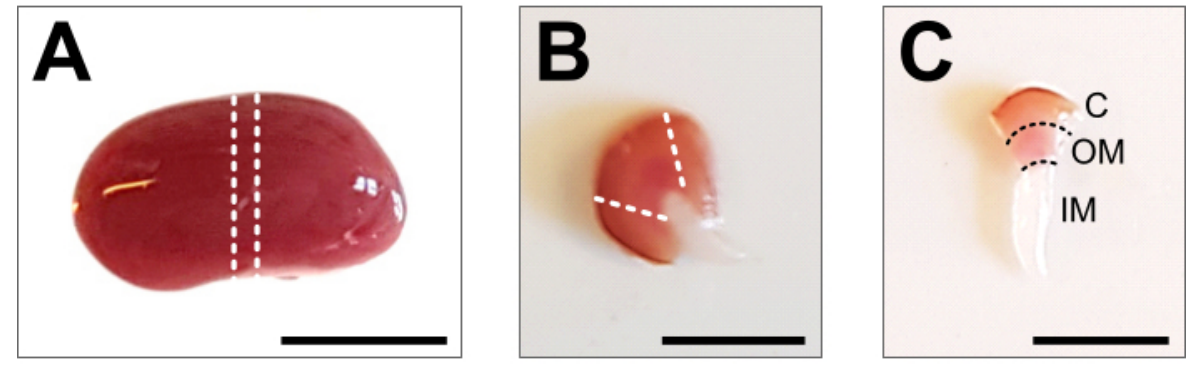

Figure 2: Kidney dissection and tissue preparation. (A) Representative image of dissected whole kidney. The dotted lines indicate the cuts required to obtain a middle slice of 1-2 $\mathrm{mm}$ with a representation of all renal cell types. (B) Representative image of obtained middle slice. The dotted lines indicate the cuts for cortex trimming from the side. (C) Representative image of central kidney piece with trimmed cortex. Cortex (C), outer medulla (OM) and inner medulla (IM) are clearly visible. Scale bar $=500 \mu \mathrm{m}$. Please click here to view a larger version of this figure. 

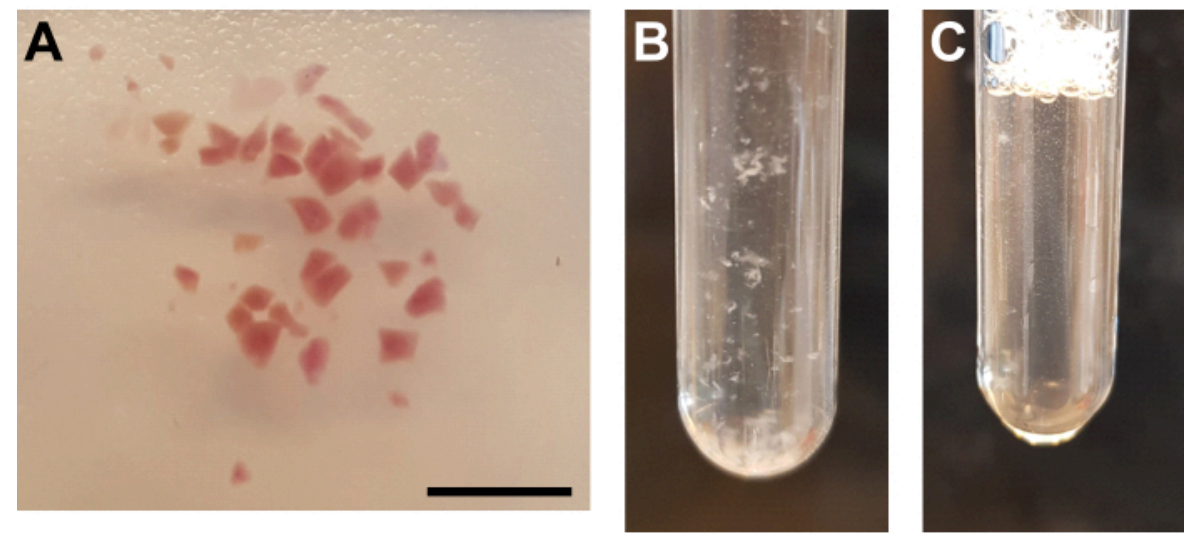

Figure 3: Tissue homogenization and nuclei purification. (A) Representative image showing sufficiently minced kidney tissue. Scale bar $=500 \mu \mathrm{m}$. (B) Homogenate after first homogenization step (25 strokes with pestle A, $2 \mathrm{~mL}$ grinder tube). (C) Homogenate after second homogenization step (15 strokes with pestle B, $2 \mathrm{~mL}$ grinder tube). Please click here to view a larger version of this figure.

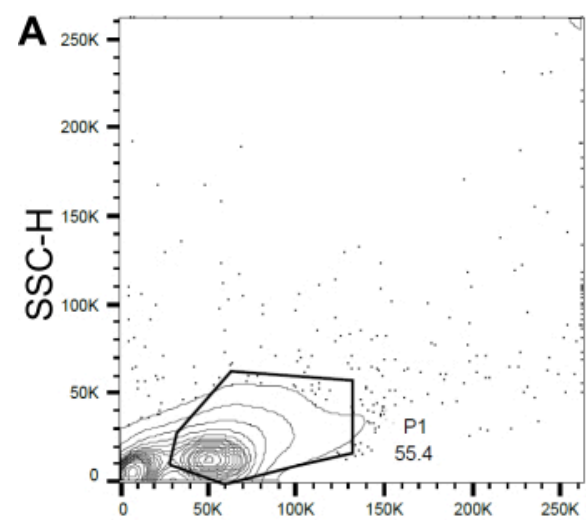

FSC-A

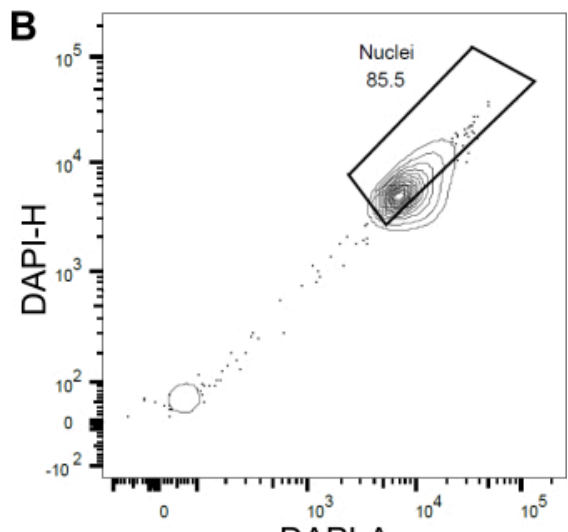

DAPI-A

Figure 4: Gating strategy for nuclei sorting. (A) A first gate P1 was set based on forward scatter (FSC) vs side scatter (SSC) to exclude debris and aggregates. (B) A subsequent gate based on DAPI-Area (DAPI-A) vs DAPI-Height (DAPI-H) excluded empty or damaged nuclei and multiplets. Please click here to view a larger version of this figure. 


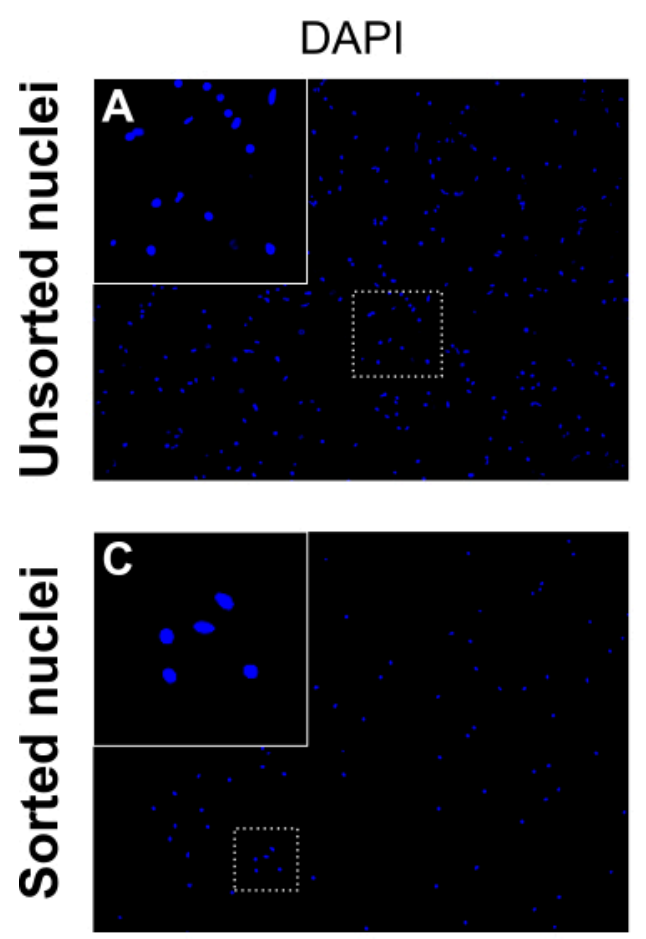

\section{Overlay (BF + DAPI)}
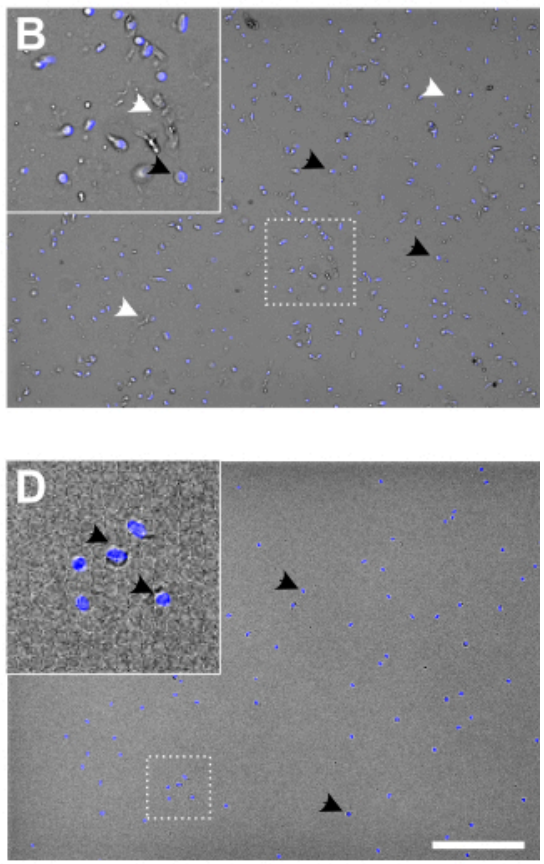

Figure 5: Nuclei suspension before and after nuclei sorting. (A, C) DAPI-stained nuclei (blue). (B, D) Overlay of DAPI and brightfield (BF) channel. Before sorting (upper panel) the nuclei suspension contains cell debris and aggregates (labeled with white arrowheads). After sorting (lower panel) the nuclei suspension appears much cleaner. Examples of DAPI-stained nuclei are labeled with black arrowheads. Good quality nuclei appear round and smooth with an intact membrane and are well-separated, whereas nuclei of poor quality appear wrinkled and show loss of the nuclear membrane. Scale bar $=250 \mu \mathrm{m}$. Please click here to view a larger version of this figure. 


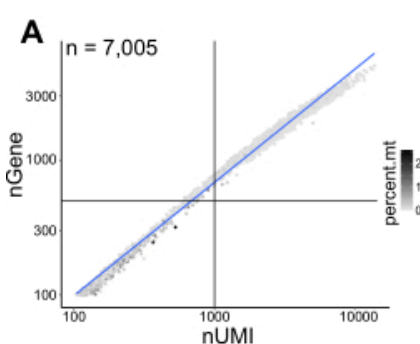

D

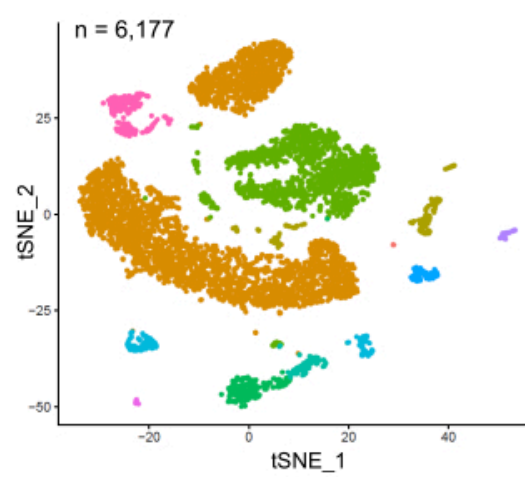

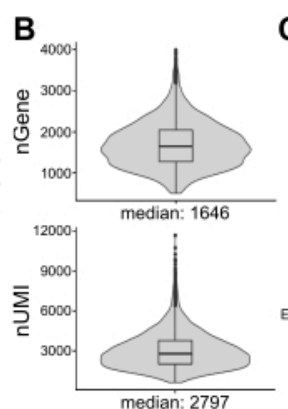

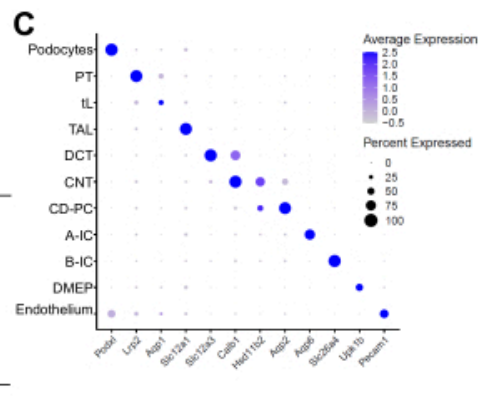

E $\quad \begin{array}{lllllll}0 & 10 & 20 & 30 & 40 & 50 \%\end{array}$

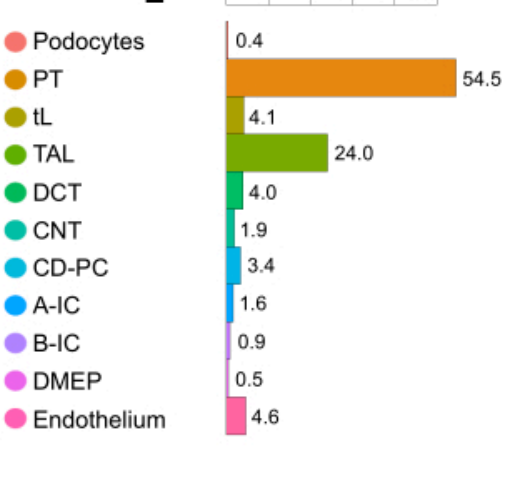

Figure 6: Quality control and analysis of a representative snRNA-seq dataset. (A) Number of genes (nGene) plotted against the number of unique molecular identifiers (nUMI) colored by the fraction of mitochondrial reads (percent.mt). Low quality nuclei correspond to the bottom left quadrant of the plot $(n=828)$ and were excluded from subsequent analysis. (B) Distribution and median of nGene and nUMI detected per nucleus in the snRNA-seq dataset, representing 6,177 nuclei (> 500 genes). Libraries were sequenced to a median depth of 8,200 mapped reads per nucleus. (C) Dot plot showing gene expression patterns of cluster-enriched markers (x-axis) for individual cell types ( $y$-axis). The size of the dot corresponds to the proportion of cells expressing the indicated gene. The color corresponds to the average expression. (D) T-distributed stochastic neighbor embedding (t-SNE) plot of identified cell types. (E) Cell type distribution in snRNA-seq dataset. PT, proximal tubule; tL, thin limb; TAL, thick ascending limb, DCT, distal convoluted tubule; CNT, connecting tubule; CDPC, collecting duct principal cells; A-IC, type A intercalated cells; B-IC, type B intercalated cells; DMEP, deep medullary epithelium of pelvis. Please click here to view a larger version of this figure. 


\begin{tabular}{|c|c|c|}
\hline Reagent & Final concentration & Volume (mL) \\
\hline \multicolumn{3}{|l|}{ (A) $4 \%$ BSA / PBS } \\
\hline Phosphate-Buffered Saline (PBS) with 10\% Bovine Albumin & $4 \%$ & 2 \\
\hline PBS (Phosphate-Buffered Saline) 1X without calcium or magnesium & - & 3 \\
\hline \multicolumn{3}{|l|}{ (B) $0.04 \%$ BSA / PBS } \\
\hline $4 \%$ BSA / PBS & $0.04 \%$ & 0.5 \\
\hline PBS (Phosphate-Buffered Saline) 1X without calcium or magnesium & - & 49.5 \\
\hline \multicolumn{3}{|l|}{ (C) Nuclei Lysis Buffer 1 (NLB1) } \\
\hline Nuclear EZ Lysis Buffer & - & 4 \\
\hline RiboLock RNase Inhibitor (40 U/ $\mu \mathrm{L})$ & $1 \mathrm{U} / \mu \mathrm{L}$ & 0.1 \\
\hline Ribonucleoside-vanadyl complex (200 mM) & $10 \mathrm{mM}$ & 0.2 \\
\hline \multicolumn{3}{|l|}{ (D) Nuclei Lysis Buffer 2 (NLB2) } \\
\hline Nuclear EZ Lysis Buffer & - & 4 \\
\hline RiboLock RNase Inhibitor (40 U/ $\mu \mathrm{L})$ & $1 \mathrm{U} / \mu \mathrm{L}$ & 0.1 \\
\hline \multicolumn{3}{|l|}{ (E) Nuclei Suspension Buffer (NSB) } \\
\hline $0.04 \%$ BSA / PBS & - & 2 \\
\hline RiboLock RNase Inhibitor (40 U/ $\mu \mathrm{L})$ & $1 \mathrm{U} / \mu \mathrm{L}$ & 0.05 \\
\hline \multicolumn{3}{|l|}{ (F) Sucrose Gradient Buffer (10 \% Sucrose) } \\
\hline \multicolumn{3}{|l|}{ Weight $1 \mathrm{~g}$ of Sucrose } \\
\hline \multicolumn{3}{|l|}{ Dissolve in $6 \mathrm{ml}$ of Nuclear EZ Lysis Buffer } \\
\hline \multicolumn{3}{|l|}{ Fill up to $10 \mathrm{~mL}$ with Nuclear EZ Lysis Buffer } \\
\hline Filter through a $0.2 \mu \mathrm{m}$ syringe filter into a fresh tube & & \\
\hline
\end{tabular}

Table 1: Solution recipes: (A) Preparation of $4 \%$ BSA/1x PBS. Filter using a $0.2 \mu \mathrm{m}$ SFCA membrane syringe filter and keep on ice until use. (B) Preparation of $0.04 \%$ BSA 1 x PBS. Filter using a $0.2 \mu \mathrm{m}$ SFCA membrane syringe filter and keep on ice until use. (C) Preparation of Nuclei Lysis Buffer 1 (NLB1). Indicated volumes are provided per sample. Keep on ice until use. (D) Preparation of Nuclei Lysis Buffer 2 (NLB2). Indicated volumes are provided per sample. Add RiboLock RNase Inhibitor to NLB2 directly before use as noted in the protocol. Keep on ice until use. (E) Preparation of Nuclei Suspension Buffer (NSB). Indicated volumes are provided per sample. Add RiboLock RNase Inhibitor to NSB directly before use as noted 
in the protocol. Keep on ice until use. (F) Preparation of Sucrose Gradient Buffer. Filter using a $0.2 \mu m$ SFCA membrane syringe filter and keep on ice until use.

\section{Discussion}

Single-cell transcriptomics advance the understanding of cell type-specific gene expression in renal physiology and disease. Here, we provided a simple and reproducible method to isolate high-quality single nuclei from frozen mouse kidney tissue for snRNA-seq in a standardized way.

For snRNA-seq, it is critical to use high-quality nuclei as input for library generation and to avoid RNA degradation during tissue processing. Therefore, the incubation of tissue pieces in RNA stabilization solution immediately after dissection is essential to protect and stabilize cellular RNA and allows to store samples at $-80^{\circ} \mathrm{C}$ indefinitely. When applying this protocol to frozen tissue without RNA stabilization solution treatment, such as archival material, a trial run is required, and the RNA quality needs to be assessed as we observed a significant loss of RNA integrity in snap-frozen tissue without prior incubation in RNA stabilization solution.

In general, appropriate sample handling is crucial to maximizing the recovery of intact, single nuclei. All resuspension steps should be carried out by pipetting carefully to avoid shear stress and physical damage. Buffers for the final nuclei resuspension and nuclei sorting should contain BSA to avoid nuclei loss and aggregation.

Buffer volumes in this protocol are optimized for very small tissue samples $(\sim 15 \mathrm{mg})$. It is critical to ensure complete cell lysis and sufficient washing to generate high-quality suspensions. Larger tissue blocks or whole kidney samples will result in excessive nuclei concentrations that lead to clumping and aggregation, high abundance of ambient RNA, and overall poor suspension quality. If larger samples or other tissues are processed, it is highly recommended to perform trial runs to determine optimal buffer volumes for minimal ambient RNA levels. Nuclei and RNA quality and concentrations need to be examined carefully as overloading results in overall poor performance.

In addition, large amounts of cell debris, causing high levels of ambient RNA not associated with single nuclei influence the sequencing results negatively. Clarification of the nuclei suspension by centrifugation through a sucrose cushion mitigates this problem to some extent, but it can also lead to bias in cell type representation by counter selecting against dense, small nuclei present, for instance, in immune cells ${ }^{16}$. If this is of concern, the sucrose gradient should be omitted. By contrast, we found that flow cytometry based on DAPI staining was critical to reduce the amount of cell debris in order to produce a high-quality single nuclei suspension.

The isolation of single nuclei has considerable advantages when compared to single-cell approaches ${ }^{8}$. It is compatible with properly frozen tissue, making the tissue collection more flexible, and circumvents the need of enzyme-based tissue dissociation, which can introduce transcriptional stress responses $^{6,17}$. Furthermore, it overcomes the dissociation bias that favors the selection of easily dissociable cell types of the renal cortex, which may lead to an underrepresentation of medullary cell types in some enzymebased approaches $5,6,10$.

Using a central kidney piece instead of whole kidney tissue further saves resources and corrects for the overrepresentation of abundant cell types as described earlier ${ }^{10}$. However, depending on the mouse model or 
phenotype investigated, it may be beneficial to use whole kidney samples instead of a single middle slice. Whole kidney samples may be more representative of true cell proportions, or changes occurring in the whole kidney, whereas a trimmed middle slice proved advantageous for medullary phenotypes or when sample material was limited. This decision, therefore, is highly user-specific and should be considered carefully.

\section{Disclosures}

The authors have nothing to disclose.

\section{Acknowledgments}

We thank the Scientific Genomics Platform at the Max Delbrück Center for Molecular Medicine in the Helmholtz Association, Berlin for technical support.

$\mathrm{JL}$ and KMSO were supported by the German Research Foundation (DFG) Research Training Group GRK 2318 and by Research Unit FOR 2841. KMSO was supported by Collaborative Research Grant 1365. AB was supported by funding from the Gottfried Wilhelm Leibniz Prize of the DFG awarded to NR.

\section{References}

1. Randall Thomas, S. Kidney modeling and systems physiology. Wiley Interdisciplinary Reviews: Systems Biology and Medicine. 1 (2), 172-190 (2009).

2. Potter, S. S. Single-cell RNA sequencing for the study of development, physiology and disease. Nature Reviews Nephrology. 14 (8), 479-492 (2018).

3. Park, J., Liu, C. L., Kim, J., Susztak, K. Understanding the kidney one cell at a time. Kidney International. 96 (4), 862-870 (2019).
4. Clark, A. R., Greka, A. The power of one: advances in single-cell genomics in the kidney. Nature Reviews Nephrology. 16 (2), 73-74 (2020).

5. Lake, B. B. et al. A single-nucleus RNAsequencing pipeline to decipher the molecular anatomy and pathophysiology of human kidneys. Nature Communications. 10 (1), 2832 (2019).

6. Wu, H., Kirita, Y., Donnelly, E. L., Humphreys, B. D. Advantages of single-nucleus over single-cell RNA sequencing of adult kidney: Rare cell types and novel cell states revealed in fibrosis. Journal of the American Society of Nephrology. 30 (1), 23-32 (2019).

7. Adam, M., Potter, A. S., Potter, S. S. Psychrophilic proteases dramatically reduce single-cell RNA-seq artifacts: a molecular atlas of kidney development. Development. 144 (19), 3625-3632 (2017).

8. Habib, N. et al. Massively parallel single-nucleus RNAseq with DroNc-seq. Nature Methods. 14 (10), 955-958 (2017).

9. Muto, Y. et al. Single cell transcriptional and chromatin accessibility profiling redefine cellular heterogeneity in the adult human kidney. Nature Communications. 12 (1), 2190 (2021).

10. Hinze, C. et al. Kidney single-cell transcriptomes predict spatial corticomedullary gene expression and tissue osmolality gradients. Journal of the American Society of Nephrology. 32 (2), 291 (2021).

11. Berger, S. L. Isolation of cytoplasmic RNA: ribonucleoside-vanadyl complexes. Methods in Enzymology. 152, 227-234, (1987).

12. Butler, A., Hoffman, P., Smibert, P., Papalexi, E., Satija, R. Integrating single-cell transcriptomic data across 
different conditions, technologies, and species. Nature Biotechnology. 36 (5), 411-420 (2018).

13. Stuart, T. et al. Comprehensive Integration of single-cell data. Cell. 177 (7), 1888-1902 e1821 (2019).

14. Park, J. et al. Single-cell transcriptomics of the mouse kidney reveals potential cellular targets of kidney disease. Science. 360 (6390), 758-763 (2018).

15. Kirita, Y., Wu, H., Uchimura, K., Wilson, P. C., Humphreys, B. D. Cell profiling of mouse acute kidney injury reveals conserved cellular responses to injury. Proceedings of the National Academy of Sciences of the United States of America. 117 (27), 15874-15883 (2020).

16. Schneeberger, $S$. et al. The neuroinflammatory interleukin-12 signaling pathway drives Alzheimer's disease-like pathology by perturbing oligodendrocyte survival and neuronal homeostasis. bioRxiv. e441313, (2021).

17. Nguyen, Q. H., Pervolarakis, N., Nee, K., Kessenbrock, K. experimental considerations for single-cell RNA sequencing approaches. Frontiers in Cell and Developmental Biology. 6108 (2018). 